INPLASY

PROTOCOL

To cite: Tian et al. The effectiveness of iron supplementation for postpartum depression: A protocol for systematic review and meta-analysis. Inplasy protocol 2020110007. doi: 10.37766/inplasy2020.11.0007

Received: 03 November 2020

Published: 03 November 2020

Corresponding author:

Zeyu Zheng

diezheng6518406@126.com

Author Affiliation:

Obstetrics Department, The

People's Hospital of

Nanchuan, Chongqing, China

Support: JMRP

(2019MSXMI01).

Review Stage at time of this submission: The review has not yet started.

Conflicts of interest:

None.

\section{The effectiveness of iron supplementation for postpartum depression: A protocol for systematic review and meta-analysis}

Tian, Y1; Zheng, Z²; Ma, C³.

Review question / Objective: To evaluate the evidence of randomized controlled trials (RCTs) for iron supplementation treatment of postpartum depression (PPD).

Condition being studied: Iron supplementation, postpartum depression, protocol, systematic review, meta-analysis.

Information sources: PubMed, Embase, Cochrane Library, Web of Science, China National Knowledge Infrastructure, China Science and Technology Journal Database and Chinese Biomedical Literature Database will be searched from their inception of databases to 31 December, 2020. The detailed search strategy for PubMed is shown in Table 1. The similar search strategies will be used for other electronic databases.

INPLASY registration number: This protocol was registered with the International Platform of Registered Systematic Review and Meta-Analysis Protocols (INPLASY) on 03 November 2020 and was last updated on 03 November 2020 (registration number INPLASY2020110007).

\title{
INTRODUCTION
}

Review question / Objective: To evaluate the evidence of randomized controlled trials (RCTs) for iron supplementation

Condition being studied: Iron supplementation, postpartum depression, protocol, systematic review, meta-analysis. treatment of postpartum depression (PPD). 


\section{METHODS}

Participant or population: Participants who meet the diagnostic criteria of PPD will be included without restrictions of nationality, age, gender, and race.

Intervention: In the treatment group, patients were given iron supplementation with no limitations of administration routes, dosage or duration of intervention.

Comparator: RCTs that have control groups with conventional treatments (such as psychological interventions and pharmacological interventions) or no treatment will be included.

Study designs to be included: All RCTs of iron supplementation for PPD will be included without language limitation. Case reports, animal experiments and reviews will be excluded.

Eligibility criteria: RCTs of iron supplementation for PPD.

Information sources: PubMed, Embase, Cochrane Library, Web of Science, China National Knowledge Infrastructure, China Science and Technology Journal Database and Chinese Biomedical Literature Database will be searched from their inception of databases to 31 December, 2020. The detailed search strategy for PubMed is shown in Table 1. The similar search strategies will be used for other electronic databases.

Main outcome(s): EPDS score will be designated as the primary outcome.

Additional outcome(s): Improvement rate will be designated as the secondary outcome.

Data management: Two reviewers will extract data from the selected studies independently. The following information will be extracted: first author's name, publication year, journal, study design, patient information, experimental intervention, control intervention, duration of follow-up, and outcomes. Any disagreement will be resolved by discussion with the third reviewer. If some important information is missing, we will contact original authors by email to request detailed information about the research.

Quality assessment / Risk of bias analysis: The Cochrane risk of bias assessment tool will be used to assess the risk of bias. Random sequence generation, allocation concealment, blinding of participants and personnel, blinding of outcome assessment, incomplete outcome data, selective reporting and other bias will be assessed by two reviews independently. A bias value of 'high', 'unclear', or 'low' will be given for each domain. The rating results will be cross-checked and the difference will be solved by the third reviewer. Bias graph and graphic summary of risk of bias will be provided in the completed review.

Strategy of data synthesis: We will perform data synthesis using Review Manager 5.3 software. Risk ratio with $95 \%$ confidence interval will be used for dichotomous variables. Mean difference with $95 \%$ confidence interval will be used for continuous variables. Heterogeneity will be examined using the 12 test. The 12 value > $50 \%$ means significant heterogeneity, and the random effects model will be used. Otherwise, the 12 value $\leq 50 \%$ means minor heterogeneity, and the fixed effects model will be utilized.

Subgroup analysis: Subgroup analysis will be performed to explore the potential heterogeneity and inconsistency based on differences in participant characteristics, administration routes, dosage and duration of intervention.

Sensibility analysis: To check the robustness and reliability of data analysis, sensitivity analysis will be applied. We will perform meta-analysis repeatedly after eliminating studies in low quality.

Country(ies) involved: China. 
Keywords: iron supplementation, postpartum depression, protocol, systematic review, meta-analysis.

Contributions of each author:

Author 1 - Yanran Tian.

Author 2 - Zeyu Zheng.

Author 3 - Chen Ma. 